\title{
EDITORIAL: HOW WORTHWHILE ARE POLLINATION NETWORKS?
}

\author{
James D. Thomson, Editor-in-Chief, Journal of Pollination Ecology \\ Department of Ecology and Evolutionary Biology, University of Toronto, Toronto, Ontario M5S 3G5, Canada
}

\author{
Journal of Pollination Ecology, \\ 28(0), 2021, pp i-vi \\ DOI: $10.26786 / 1920-$ \\ $7603(2021) 652$ \\ *Corresponding author: \\ james.thomson@utoronto.ca
}

\begin{abstract}
An occupational hazard for editors is the powerful temptation to editorialize. In pondering a possible contribution to the SCAPE Special Issue, I decided to indulge that temptation by expanding part of my plenary address to SCAPE 2018 at Abisko into a personal comment expressing scepticism about the practice of constructing pollination networks. At that time, and on various occasions since, some colleagues suggested that publishing my perspective might help foster a useful conversation.
\end{abstract}

The pollination network approach has become extremely popular. Its promise to encompass "the entire community" is surely a worthy and ambitious goal, but my personal experience left me sceptical from my first exposure to the approach. I must state at the outset that I am not an authority on network construction or interpretation; I have not followed the literature with any care. This note is nothing like a meta-analysis or a formal critique (for the latter, see Blüthgen 2010). I cite hardly any references, because I am not targeting specific papers. I expect that few of my points are new. I am simply trying to explain the origin of my personal scepticism by developing an analogy between network studies and an earlier fad of niche-based studies. I offer the analogy because I consider it both strong and sobering.

Along with many others in my cohort of graduate students in the early 1970s, I posed dissertation questions that embraced the prevailing notion that quantifying niche overlaps was a cornerstone of a community ecology based on interspecific competition. But as my attention shifted from the library to the field, I came to regard my earlier enthusiasm as insufficiently critical. Although my dissertation research gave me several insights into pollination biology, the insights that have lasted through my career arose in spite of, rather than because of, the fad for niche metrics. And now, at the other end of my career, I see the new enthusiasm for networks as echoing the old enthusiasm for niche metrics. In both cases, seductive techniques promised more than they can deliver. Regarding key assumptions, optimism trumped wariness.

\section{A BACKWARD-LOOKING CARICATURE OF NICHE-BASED COMMUNITY ECOLOGY}

The enthusiasm for niche metrics arose from the adoption of the multidimensional Hutchinsonian niche, along with a belief in the centrality of Gausian processes such as competitive exclusion, limiting similarity, and resource partitioning. MacArthur's paper on the population ecology of warblers had already made it into the textbooks. His "birds in trees" picture became a touchstone of the approach. Although MacArthur presented a variety of perspectives and data, the "take-home message" for too many readers boiled down to the idea that foraging in different zones of trees was what allowed these different species of ecologically similar birds to coexist, i.e., that overlap in resource use was a determinant of population dynamics. The intoxicating implication was that one could "explain" community composition by collecting easily observed behavioural data. At the deepest level of oversimplification, pairwise niche overlaps (typically denoted as $a_{i j}$ ) were seen as related to the $\alpha_{i j}$ competition coefficients of the multispecies Lotka-Volterra models of interspecific competition. Stable, equilibrial communities were hypothesized to present detectably non-random patterns of resource use. Many dissertations were launched in search of the niche overlap patterns that offered such explanatory power. 
That hope was too good to be true. Many factors, including disturbance, predation, disease, and metapopulational dynamics conspire to prevent communities from reaching competitive equilibria. Some critics argued that competition could be revealed only by manipulative experiments; others used null models to question numerous "patterns" of resource use that had previously been ascribed to resource partitioning. For an example from pollination biology, Poole \& Rathcke (1979) used a null model to dismiss a suggestion by Stiles that a guild of hummingbirdpollinated plants displayed regularly spaced times of peak flowering, suggestive of competition for pollinators. It became apparent that niche overlaps were not a panacea for understanding community structure. Overlaps were not alphas. By the early 1980s, Simberloff (1982) felt justified in offering the bleak assessment that competition theory had "caused a generation of ecologists to waste a monumental amount of time."

In designing my own dissertation, I also focused on the zeitgeist-y possibility that zoophilous plants might compete for pollination services, and that such competition might result in detectable patterns at the whole-community level. I felt that pollination relationships were especially suited for studies of niche overlap: faunal overlaps between plant species could be estimated by observations of flower visitors, and temporal overlaps could be calculated from floral census data. Critically, I could also estimate pollination success, via fluorescent dye transfer, to determine the extent to which pollination service was reduced by greater overlap from other species. For the subalpine meadow habitats I studied, the deflating conclusion (Thomson 1982) was that the amount of overlap experienced by a plant population bore no relationship whatsoever to my estimates of visitation rate. My overlap measures did successfully indicate the extent to which pairs of plants shared a conceptual ecological "location" that included pollinator sharing and coincidence in blooming time, but proximity in that space had no bearing on pollination success. Overlaps did not even determine proximate measures of reproductive success, let alone ultimate population trends.

Why was this so? At the most fundamental level, many factors can act to undermine the simplistic assumption that ecological similarity induces negative, competitive interactions. Pollinator activity levels and plant affiliations vary temporally through the season, and spatially within habitats. Patchiness is ubiquitous. I am still working on one specific spatial process, the "magnet species" phenomenon by which an attractive species helps, rather than harms, a less attractive neighbour by drawing more pollinators in to a local area. Magnet effects can also show temporal holdover, if animals continue to show site fidelity after an attractive plant has finished blooming (Ogilvie \& Thomson 2016). And if a species does experience magnet effects, the advantage of higher visitation may be offset by heterospecific pollen deposition (Thomson et al. 2019). Given this diverse portfolio of effects, it is unsurprising that naïve measures of ecological similarity fail to capture the consequences of plantplant interactions for pollination. The net effects could be positive, negative, or effectively neutral; they could vary spatially across the habitat; and they could change sign over time.

\section{THE ANALOGY WITH POLLINATION NETWORKS}

The two activities of constructing a bipartite network and constructing a set of pairwise niche overlaps begin with the same data set, specifically a matrix of plant species $\times$ pollinators. Formally, both avenues of analysis can be considered to be elaborate numerical transformations of the original data matrix. In niche analysis, the matrix is considered to be imbued with negative, competitive interactions between plant species, based on similarity of shared pollination; in network analysis, the same matrix is imagined to reflect beneficial, mutualistic interactions between plants and pollinators. The network perspective leads to the calculation of different summary statistics, such as connectance and nestedness. Such indices strike me as rather abstract and abiological, but they are arguably useful and meaningful to skilled interpreters, especially in the context of comparing the properties of different matrices. Network papers typically contain elaborate diagrams that use lines to show which plants are linked with which animals. I have repeatedly heard practitioners praise such diagrams as beautiful. I find them tediously impenetrable, but this may be a case where beauty lies in the eye of the beholder. What I consider 
more worrisome are extrapolations from such indices to conservation-related conclusions regarding the persistence of species or "the stability of networks." Like some of the dubious interpretations of niche overlap, such extensions depend on a strong link between the pollination interaction and the dynamic maintenance of populations. Unfortunately, few such links have been demonstrated. Furthermore, just as overlapping in pollinator niche space can be beneficial rather than harmful, so can getting visited by a particular animal in a network; visitors that remove large amounts of pollen and deliver small amounts can be beneficial mutualists in some contexts but detrimental pollen thieves in others (Thomson 2003). Treating a plant $\times$ pollinator matrix purely as a gallery of mutualistic benefaction is unrealistic. On top of those difficulties, interactions in a bipartite network will similarly be influenced by spatial phenomena, such as magnet species effects, site holdover, and the deposition of heterospecific pollen. These factors will usually take effect at smaller spatial scales than the scale at which the network is characterized. Network connections will also shift during the season, as different plants come into bloom and insect populations rise and fall. Therefore, individual plants will typically encounter a localized and dynamic pollination experience different from that summarized in the network. Heraclitus famously proposed that one cannot step into the same river twice. Pollination communities are river-like in this respect. They will similarly elude one-time characterizations, whether by network metrics or any other static summary.

\section{CONCLUSION AND SOME RECOMMENDATIONS}

The currently fashionable practice of interpreting a plant $\times$ pollinator matrix as a catalogue of mutualisms suffers from some of the same shortcomings as the formerly fashionable practice of reading it as a chronicle of competitive struggle. Both rest on assumptions that the matrix elements represent a homogeneous set of a single type of biological interaction. But clearly established counterexamples reveal such assumptions to be inadequate. I suggest that inferring dynamic properties such as "network stability" from such a matrix is seldom if ever justified. The process of compiling such a matrix is certainly a valuable part of any investigation into the pollination ecology of a community. It is always good to know who is visiting what, but I would argue that the best use of that matrix is to launch specific testable hypotheses, rather than apotheosizing the matrix into a network and calling the job finished. Ideally, those hypotheses would then be subjected to replicated observations and experiments that (1) actually determine the fitness consequences of the interactions and (2) take into account the temporal and spatial variation that characterizes all real communities. In analysing the matrix itself, investigators should not bypass classical multivariate approaches that have served dependably as "data reduction techniques" in community ecology. Ordinations were designed to extract underlying compositional tendencies from sprawling data sets that were too unwieldy to reveal their secrets to mere inspection. In contrast, those nearly ubiquitous network diagrams do nothing to distill or digest data; they merely re-express the entire content of the matrix in a different format. It reminds me of my childhood technique of pushing vegetables around on my plate to imply that I had been eating them.

Diagrams aside, network summary statistics do distil a raw data matrix into manageable numbers, but their usefulness is limited. For example, connectances can in principle be compared across different studies, but such comparisons are tricky. As Paine (1988) showed for food webs, connectance values depend on investigators arbitrary decisions about spatial scale and the pooling of taxa for convenient identification. Because such pooling is nearly universal in studies of pollinating insects (e.g., "small halictids" or "Bombus terrestris/lucorum group"), connectances cannot be compared across studies unless the studies used the same criteria. Agreeing on a standard set of pooling rules could improve comparability, but would require levels of collegiality and coordination that are seldom seen in community ecology. The dependence of connectance on taxonomic pooling also prevents useful comparison of observed values to universal standards, such as $50 \%$ connectance. The intensity of sampling would also be important, as more observations would inevitably add more links. Sampling limitations have been treated in depth by Blüthgen (2010) and doubtless by others since. The essential point is that pollination networks are 
often presented as innate properties of systems, but they are really more like short, blurry video clips from a particular place over a particular stretch of time. Considering these limitations, I think that the best use of connectance in pollination communities would be for a single investigator to track how communities change through succession, or in response to perturbations, adhering to a rigid sampling protocol. Still, it takes so much effort to assemble even one network that the necessity for replication is daunting indeed-and extensive replication would be needed to distinguish meaningful signal above the noise of the river.

Time and again, science is rescued by its cardinal virtue: it is self-correcting. Nevertheless, it remains susceptible to passing fads and fashions. A network approach seems valid and rigorous for data sets that conform to the fundamental nodesand-links assumption of graph theory, but interactions in real pollination systems are not that simple. Assuming otherwise risks a slide into wishful thinking.

\section{REFERENCES}

Blüthgen N (2010) Why network analysis is often disconnected from community ecology: A critique and an ecologist's guide. Basic and Applied Ecology 11:185195.

Ogilvie JE, Thomson JD (2016) Site fidelity by bees drives pollination facilitation in sequentially blooming plant species. Ecology 97:1442-1451.

Paine RT (1988) Road maps of interactions or grist for theoretical development? Ecology 69:1648-654.

Simberloff D (1982) The status of competition theory in ecology. Annales Zoologici Fennici 19:241-253.

Thomson JD (1982) Patterns of visitation by animal pollinators. Oikos 39:241-250.

Thomson JD (2003) When is it mutualism? American Naturalist 162:S1-S9.

Thomson JD, Fung HF, Ogilvie JE (2019) Effects of spatial patterning of co-flowering plant species on pollination quantity and purity. Annals of Botany 123:303-310. 